Courrier du Centre international Blaise Pascal

$9 \mid 1987$

Varia

\title{
Yoichi Maeda 1911-1987
}

Jean Mesnard

\section{OpenEdition}

Journals

Édition électronique

URL : http://journals.openedition.org/ccibp/447

DOI : $10.4000 /$ ccibp 447

ISSN : 2493-7460

\section{Éditeur}

Centre international Blaise Pascal

\section{Édition imprimée}

Date de publication : 4 septembre 1987

Pagination : 3-6

ISSN : 0249-6674

Référence électronique

Jean Mesnard, « Yoichi Maeda 1911-1987», Courrier du Centre international Blaise Pascal [En ligne]

9 | 1987, mis en ligne le 07 décembre 2015, consulté le 20 avril 2019. URL : http://

journals.openedition.org/ccibp/447 ; DOI : 10.4000/ccibp.447

Ce document a été généré automatiquement le 20 avril 2019.

Centre international Blaise Pascal 


\title{
Yoichi Maeda 1911-1987
}

\author{
Jean Mesnard
}

1 Loin de condamner au repli sur soi, une vie d'étude peut se prêter à des expériences humaines extraordinaires. Pour l'auteur de ces lignes, la recherche sur Pascal a été l'occasion de rencontres mémorables, enrichissement pour l'esprit associé au charme de l'amitié. Entre tous les visages ainsi apparus, dont beaucoup, hélas! Se sont effacés, celui de Yoichi Maeda garde, dans le souvenir, un relief exceptionnel.

2 Avant de le connaître, il m'avait été donné, en 1962, dans le recueil collectif Pascal et PortRoyal, de dire mon admiration pour la part, malheureusement très limitée, de son œuvre qui était alors accessible au public français. La première rencontre, à Paris, en 1965, suivie, en novembre 1966, d'une visite à Bordeaux et d'une excursion en commun au château de Montaigne, procura l'émerveillement de découvrir l'homme au-delà de l'auteur. Dès lors les relations étaient solidement nouées. Elles s'entretenaient tout naturellement, grâce à de fréquents voyages, de l'un en France, de l'autre au Japon, et par le moyen d'une correspondance fidèle.

Dans sa conversation savoureuse et passionnante, toujours débordante de vie, Yoichi Maeda évoquait volontiers ses recherches. Mais il aimait aussi revenir sur son passé, non par complaisance en soi, mais par une claire conscience de l'originalité de son destin et pour en dégager une réflexion qui dépassait de beaucoup sa personne.

Dans ses origines familiales et dès ses premières années, il avait trouvé les conditions les plus propices à l'affirmation des deux traits qui frappaient d'abord en lui: son enracinement dans la tradition japonaise, son ouverture au monde occidental. Né quelque cinquante années après le début de l'ère Meiji, le 3 novembre 1911, dans la préfecture de Gunma, c'est-à-dire en plein pays montagneux, au Nord de Tokyo, au cœur de ce qu'on pourrait appeler le Japon profond, il n'en fut pas moins, d'emblée, plongé dans la vie moderne et attiré par les vastes horizons. Son père, Taemon Maeda, auquel il se référait souvent comme à un modèle, était un lettré nourri de sagesse orientale, un digne représentant de la tradition civile - à tous les sens du mot - du Japon, pendant, et parfois opposé de sa tradition militaire. Curieux de l'Occident, et passionné par les questions religieuses, il avait adhéré au protestantisme et s'était enthousiasmé pour les Quakers, 
dont il retenait surtout l'esprit de paix et le sens de la fraternité. Ainsi faisait-il régner autour de lui une haute aspiration à l'humain et à l'universel.

5 Appartenant à la haute administration japonaise, Taemon Maeda fut, au lendemain de la première guerre mondiale, ministre de l'Éducation Nationale. En 1923, chargé d'une mission diplomatique à Genève, il y emmena sa famille et fit étudier son fils dans une école internationale, où l'enseignement $d u$ français tenait évidemment la première place. Ainsi s'éveilla la vocation qui devait porter le jeune homme vers les études de langue et de littérature françaises. Une vocation qui se confirma et s'enrichit au cours de brillantes années passées à l'Université de Tokyo, jusqu'en mars 1934.

\section{Yoishi Maeda}

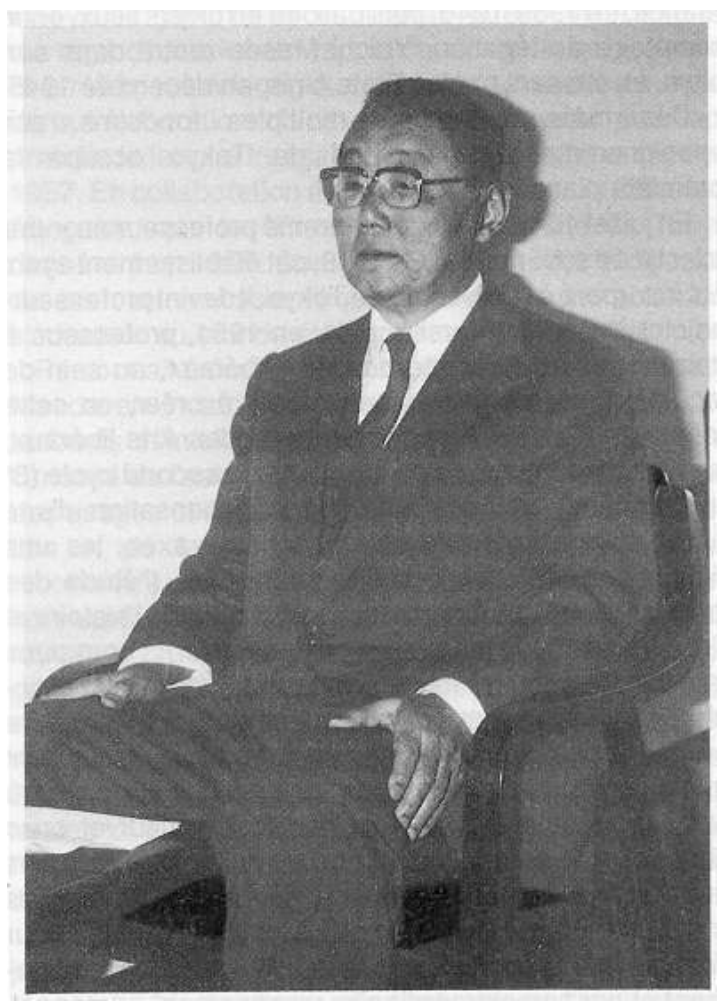

Cliché Madame Maeda

En octobre de la même année, l'étudiant japonais, devenu boursier du gouvernement français, entamait un long séjour à Paris. Sa prédilection pour Pascal, l'un des écrivains occidentaux les plus profondément assimilés par la culture de son pays, s'était désormais affirmée. Il eut la chance, à la Sorbonne, de pouvoir préparer sa thèse de doctorat sous la direction de Léon Brunschvicg. Le sujet choisi, Les Arguments apologétiques chez Montaigne et chez Pascal, permettait de rigoureuses confrontations de textes, entre l'Apologie de Raymond Sebond et les Pensées, sans oublier l'Entretien avec M. de Sacy. Il donnait lieu aussi de reprendre, sous un angle neuf, l'étude du dialogue exemplaire entre deux esprits dont les affinités ne sont pas moins saisissantes que les oppositions. L'ouvrage fut achevé en 1940 et présenté à l'Université de Paris. Il obtint, au mois d'octobre, le permis d'imprimer. Mais les contraintes de cette sombre époque empêchèrent l'accession à soutenance. C'est en mars 1947, devant l'Université impériale de Tokyo, que fut conquis, avec la même thèse, le grade de docteur ès Lettres. L'ouvrage fut publié dans une version japonaise en 1949. De la rédaction originale, il subsiste quelques exemplaires multigraphiés. Deux 
morceaux en ont été publiés en France, l'un, relatif à l'Entretien avec M. de Sacy, dans le recueil Écrits sur Pascal, procuré par Louis Lafuma en 1959, l'autre, consacré à un parallèle entre l'Apologie de Raymond Sebond et les Pensées, dans le Bulletin de la Société des Amis de Montaigne, en 1961. Ayant suivi quelque temps la carrière diplomatique, d'abord comme attaché à l'ambassade du Japon en France, en 1939-1940, puis ballotté en divers lieux, enfin secrétaire de légation, Yoichi Maeda rentra dans son pays, en passant par les ÉtatsUnis, en décembre 1945. Désormais, entre ses multiples fonctions, son enseignement à l'Université de Tokyo occupa la première place.

7 En juillet 1945, il avait été nommé professeur au « premier lycée supérieur ». En 1949, cet établissement ayant été incorporé à l'Université de Tokyo, il devint professeur adjoint à cette Université, puis, en 1951, professeur. Il appartenait à la Faculté des Arts libéraux, au sein de laquelle il contribua très activement à créer, en cette même année 1951, un département des Arts libéraux, assurant la formation des étudiants de second cycle (39 et 49 années). L'objectif visé était la dispensation d'une haute culture, orientée selon plusieurs axes, les uns définis par quelques grandes civilisations (l'étude des langues était ainsi étroitement unie à celle de l'histoire et de la vie sociale), les autres par les secteurs communs que constituaient les relations internationales, la philosophie et l'histoire des sciences. Remarquable structure pour l'élaboration d'un humanisme moderne, accueillant aux diversités et soucieux de l'unité. Heureux pays où une Université peut se réformer de l'intérieur et sous l'impulsion de ses maitres! Conçues de la manière la plus large et la plus compréhensive, les études françaises s'inséraient ainsi, par surcroît, dans un cadre qui leur donnait tout leur relief. Par la qualité de son enseignement et par son extraordinaire rayonnement personnel, Yoichi Maeda fit briller d'un éclat très vif la section "Civilisation et Société en France " qu'il dirigea dès l'origine et jusqu'à sa retraite, en mars 1972. Son rôle fut d'autant plus efficace qu'il eut à former, pendant plus de vingt ans, une bonne part des élites du Japon. Ses disciples ont peuplé les Universités japonaises - et quelquefois étrangères - s'imposant dans des spécialités variées, la littérature française, évidemment, mais aussi la littérature comparée, l'histoire du théâtre, l'histoire de l'art, la philosophie. Parallèlement, d'autres se distinguaient dans la diplomatie, dans la haute fonction publique, ou parmi les cadres des entreprises privées. Que de services japonais, en France et à l'étranger, où le nom de M. Maeda éveille d'heureux souvenirs et facilite merveilleusement les démarches! Dans son enseignement, rien ne lui était plus cher que son séminaire sur Pascal. Lieu d'exposition pour les recherches achevées, de mise à l'épreuve pour les hypothèses nouvelles, de réflexion sur des textes dont l'étude érudite n'avait d'autre objet que d'enrichir la signification humaine, enfin de formation pour les disciples les plus proches, ceux qui ont aujourd'hui pris le relais du maître et poursuivent son œuvre.

8 Le séminaire portait essentiellement sur les Pensées. Il en allait de même pour l'œuvre qui se développait en parallèle. De part et d'autre, régnait la même exigence de rigueur. Les auditeurs du séminaire avaient toujours sous les yeux la photographie du fragment original qui faisait l'objet de la leçon. De même, dans le bureau de sa petite maison où il passa tant d'heures studieuses, Yoichi Maeda ne travaillait jamais sans avoir à portée de la main la fameuse phototypie Brunschvicg. À quoi s'ajoutaient les microfilms des deux Copies, qu'il avait commandés à la Bibliothèque Nationale : car ce disciple de Brunschvicg avait bien vite compris la portée décisive des découvertes de Tourneur et Lafuma. De plus, au séminaire comme dans l'œuvre personnelle, l'étude des fragments prenait appui sur toutes les éditions publiées depuis le XVII e siècle, sur leurs divers classements, sur les 
interprétations successives qu'elles avaient proposées. Pascal, héritier de la Bible et de la sagesse païenne, de saint Augustin et de Montaigne; Pascal, avec lequel, pendant trois siècles, beaucoup de grands esprits avaient entretenu un commerce amical ou conflictuel; Pascal fournissait une clé précieuse pour entrer dans toute la culture occidentale. L'extrême précision allait de pair avec les plus amples perspectives.

Dans sa thèse, Yoichi Maeda avait déjà fort bien tenu ces deux bouts de la chaîne, situant l'un par rapport à l'autre Montaigne et Pascal avec finesse et profondeur, et faisant jaillir la lumière de rigoureuses confrontations de textes: il montrait en particulier dans l'Entretien avec M. de Sacy, connu apparemment de seconde main par les Mémoires de Fontaine, un écrit authentique de Pascal. L'œuvre se poursuivit, en 1966, par une traduction japonaise des Pensées, opérant un choix remarquable en faveur du classement Lafuma. Mais elle trouva son centre dans une entreprise de grande envergure, un commentaire détaillé des célèbres fragments, toujours selon l'ordre de l'édition Lafuma. À partir de 1960, ce commentaire fut publié régulièrement dans la revue Kokoro. Continué jusqu'en 1981, il comptait alors 160 articles. Encore ne couvrait-il que les sept premières liasses du classement, soit les numéros 1 à 131 de Lafuma (sur un millier). Beau témoignage de la science de l'auteur, du dynamisme de l'éditeur, de l'appétit de culture des lecteurs!

10 À ce commentaire, il fallait une idée directrice. Elle fut exposée dans un article fondamental, en 1964 dans les Études de langue et de littérature françaises, sous le titre Le premier jet du fragment pascalien sur les deux infinis, et dont la teneur fut reprise plusieurs fois, notamment dans les Chroniques de Port-Royal, en 1972, et dans le recueil Méthodes chez Pascal, en 1979. À propos du fragment sur les deux infinis, Yoichi Maeda proposa, des nombreuses corrections portées sur les autographes des Pensées, une interprétation nouvelle de portée décisive. Les variantes ne sont pas, sauf rares exceptions, à considérer une à une, comme si chacune d'elles avait une fonction particulière. Elles répondent à un dessein systématique de révision, exécuté un certain temps après la rédaction originale, et en une seule fois. Éliminer ou retenir les corrections conduit ainsi à isoler deux versions successives, et permet de procéder à deux lectures distinctes, chacune pourvue de sa propre cohérence. Nombre de pensée, et parmi les plus importantes, prennent alors un relief nouveau, parce qu'elles se prêtent à une étude génétique. Aux manuscrits des Pensées Yoichi Maeda avait arraché un nouveau secret. Du problème du classement, si brillamment éclairé par Louis Lafuma, il ramenait l'attention à celui du texte et de son analyse.

11 Comme jamais chez lui l'érudition ne faisait perdre de vue la culture, les années où s'élaborait ce système de lecture voyaient aussi paraître deux ouvrages d'initiation destinés au grand public japonais : en 1968, Pascal : ce que signifie le « roseau pensant »; en 1969, Pascal et notre temps.

$12 \mathrm{Au}$ cours de ses dernières années, malgré plusieurs épreuves de santé, Yoichi Maeda s'appliqua plus assidûment que jamais à la poursuite de son commentaire et à sa publication en une série de volumes. Deux premiers volumes virent le jour en 1980 et 1985, superbement présentés. Un troisième, posthume, mis au net par des disciples fidèles, viendra bientôt s'y joindre. Mais que de chemin resterait à parcourir pour mener l'entreprise à son terme! Nous n'en possédons pas moins là le modèle d'une édition commentée des Pensées unique en son genre. L'édition proprement dite est parfaitement accessible au public français. Chaque fragment s'y présente sous trois formes successives : photographie de l'original, transcription «figurée» respectant l'orthographe, la 
ponctuation, la disposition du manuscrit, et distinguant éventuellement les rédactions successives, puis nouvelle transcription selon l'usage moderne. Le commentaire en japonais, amplement développé, s'attache d'abord à l'explication littérale; il vise naturellement à situer le texte dans son époque, élucidant les allusions historiques, marquant le rapport avec les sources; il rappelle et critique les commentaires antérieurement proposés; enfin il dégage l'intérêt permanent du fragment, veillant en particulier à en faire saisir la portée pour le lecteur japonais, ce qui conduit à une riche confrontation entre l'Orient et l'Occident. De nouveau, l'érudition débouche sur la culture.

13 Aussi bien l'œuvre pascalienne de Yoichi Maeda ne peut-elle se séparer de ses autres activités. À l'auteur qui ne cessait de féconder son esprit, il demandait les principes d'une meilleure intelligence des problèmes contemporains qui le sollicitaient: celui de la civilisation japonaise, celui de l'Éducation et de l'Université, celui des relations internationales et du dialogue des cultures, celui du rapport Orient-Occident.

Il avait appris très tôt à connaître le monde. Satisfaction d'une curiosité, mais aussi appel à la réflexion. Les années de jeunesse passées en France lui inspirèrent un petit ouvrage : En étudiant en Occident (1953). Attiré aussi par les États-Unis, comme tout le Japon d'aprèsguerre, il s'y rendit à maintes reprises et composa sur ce pays un autre livre de souvenirs : En faisant la tournée des Universités américaines (1961). Il fut invité en particulier, à l'Université des îles Hawaii, à participer aux travaux d'un Centre d'Études sur les relations Orient Occident. Il est naturel qu'il ait été appelé, en 1963, au Comité japonais de l'UNESCO, dont il devint vice-président en 1970.

15 Au Japon, il fait beaucoup pour la diffusion et le prestige de la langue française. Il avait été précepteur du prince héritier pour cette langue, et il aimait rappeler le choix qu'il avait fait, pour former son illustre élève, des Aventures de Télémaque. L'idée d'une royauté associée à une haute exigence morale et spirituelle correspondait parfaitement à son propre idéal. Parallèlement, il avait été chargé d'un cours de français à la NHK (Radio Télévision japonaise) et il avait publié en 1954 un ouvrage d'initiation en rapport avec cet enseignement. Une réflexion pédagogique originale se manifesta aussi dans l'élaboration d'une méthode nouvelle pour apprendre la langue française, publiée en collaboration en 1957. En collaboration aussi une Histoire de la littérature française, que les Presses de l'Université de Tokyo donnèrent en 1955. Comme le prouvait déjà la diffusion de son œuvre scientifique, il savait admirablement s'adresser au monde étudiant et au public cultivé.

Personnalité éminente du Japon contemporain, Yoichi Maeda en incarnait à merveille les traits les plus attachants : l'intelligence, la puissance d'assimilation, la curiosité de l'autre et le désir d'en apprendre, le respect des usages et l'esprit de concorde, l'alliance de la tradition et de la modernité. Ses compatriotes ont su reconnaître ses grands mérites par les charges et les honneurs dont ils l'ont gratifié. À peine avait-il pris sa retraite de l'Université de Tokyo qu'il devenait directeur général de la Maison Internationale du Japon (1972). En 1981, il prit la direction de la Bibliothèque centrale de la ville de Tokyo et la présidence du Conseil National des Bibliothèques publiques. Les décorations les plus flatteuses lui étaient accordées. En 1981, il reçut le prix de l'Institut du Japon; en 1984, celui de la Fondation du Japon.

17 À l'étranger, son double rôle de témoin de la culture japonaise et de la culture française, complété par une vive curiosité pour le monde anglo-saxon, l'avaient souvent fait distinguer. En 1967, il avait obtenu le doctorat honoris causa de l'Université de Michigan. 
Quant à la France, elle avait toujours su apprécier en lui un ami sûr et efficace, dont l'œuvre et l'activité lui faisaient honneur. Il était un membre éminent de la Société des Amis de Port-Royal, devant laquelle il prononça de brillantes conférences en 1970 et 1973. L'un des animateurs du colloque de Clermont-Ferrand de 1976, il fut aussi l'un des membres fondateurs du Centre International Blaise Pascal. La capitale de l'Auvergne, qui lui était naturellement chère, reçut encore sa visite en l'automne 1980, lors du colloque sur les sœurs de Pascal. Titulaire des Palmes académiques dès 1954, il avait reçu en 1966 les insignes de chevalier de la Légion d'Honneur. Invité en 1970 à l'Université de Bordeaux pour participer au jury de la thèse d'un de ses disciples, il fut, pendant l'année universitaire 1972-1973, professeur associé à l'Université de Paris-Sorbonne. De la même Université il était fait docteur honoris causa en février 1980. Au début de novembre 1987, l'Académie française lui décernait le Prix de la Francophonie. Choix exceptionnellement flatteur que celui d'une personnalité non francophone comme bénéficiaire de cette haute récompense, mais choix profondément juste, puisqu'il couronnait toute une vie d'intimité avec la culture française et de défense de ses valeurs à l'échelle mondiale. Hélas! Yoichi Maeda ne put recevoir cette heureuse nouvelle qu'au moment où s'aggravait dangereusement une maladie de cœur dont il souffrait depuis plusieurs années. La joie de ses amis fit bientôt place à une grande tristesse lorsqu'ils apprirent sa disparition, survenue le 22 novembre.

Devant un Japonais, si riche, si ouverte que soit sa personnalité, un Français ne peut échapper à l'impression d'un certain mystère. Chez Yoichi Maeda, la franchise, la simplicité, la jovialité n'allaient pas sans beaucoup de pudeur. Il n'est pourtant pas impossible de deviner à quoi son cœur était le plus attaché.

Non pas, assurément, aux honneurs qui lui furent si abondamment et si légitimement dispensés : il en parlait peu et ne songeait nullement à s'en glorifier. Non pas à l'autorité dont il jouissait en tant de lieux et au pouvoir qu'elle lui procurait : il ne s'en servait qu'en faveur de causes désintéressées.

Il était attaché à son métier. Un métier à trois faces, dont chacune lui était aussi chère : l'enseignement supérieur, les relations culturelles, la recherche pascalienne. Quelle force l'entraînait dans cette triple tâche ? Était-ce une sorte de passion? Le mot ne serait pas tout à fait exact. Car ce qui s'imposait chez lui, c'était la lucidité, la maîtrise, l'aisance réfléchie avec laquelle il dominait ses entreprises, la conviction raisonnée qui l'animait. Aussi bien l'homme de pratique, amoureux du travail bien fait, était-il toujours relayé chez lui par l'homme de pensée, constamment appliqué à redéfinir finalités et méthodes dans les champs d'exercice de son activité. Entre la personne et l'œuvre, l'unité était totale.

21 Mais, plus profondément qu'à cette œuvre, c'est aux êtres que son cœur était attaché.

22 À sa famille. Qui n'a pas apprécié, à ses côtés, la discrétion, le dévouement associés à la riche personnalité de $\mathrm{M}^{\mathrm{me}}$ Maeda ? Outre un fils disparu prématurément dans un accident de montagne - grand chagrin dans une vie pour le reste si comblée - il eut deux filles, auxquelles il avait su transmettre son amour de la langue française et sur lesquelles sa conversation revenait souvent.

23 À ses disciples. L'une de ses grandes joies fut de trouver parmi ses élèves des esprits supérieurement doués dont la vocation se dessina dans la ligne de la sienne. À l'Université de Tokyo, dans le dialogue entre la France et le Japon, dans le domaine des études 
pascaliennes, l'œuvre féconde dont il avait été l'initiateur a pu se poursuivre sous ses yeux, lui apportant la garantie précieuse qu'une sorte de survie lui était promise.

24 À ses amis. Le cercle de ceux qui, pour lui, méritaient véritablement ce nom était sans doute assez restreint. Sa cordialité naturelle excluait toute familiarité de surface. Mais à ceux qu'il avait choisis - parmi eux plusieurs Français - il prodiguait le meilleur de luimême : en sa compagnie, le plaisir n'allait jamais sans le profit. Il est difficile d'oublier la simplicité délicate et chaleureuse de son accueil dans sa demeure quasi champêtre de Setagaya-ku à Tokyo, ou dans sa maison de villégiature de Karuizawa, au milieu des arbres. Dans ces cadres intimes se révélait l'essence de l'homme, où la supériorité de l'esprit s'alliait à une sensibilité dénuée de tout artifice, très proche de la nature et de la vie.

INDEX

Mots-clés : Maeda (Yoichi)

\section{AUTEUR}

\section{JEAN MESNARD}

Professeur émérite de littérature française du XVIIe siècle

Université de Paris-IV 